\title{
DIRAC-SOBOLEV INEQUALITIES AND ESTIMATES FOR THE ZERO MODES OF MASSLESS DIRAC OPERATORS
}

\author{
A. BALINSKY, W. D. EVANS, AND Y. SAITŌ
}

\begin{abstract}
The paper analyses the decay of any zero modes that might exist for a massless Dirac operator $H:=\alpha \cdot(1 / i) \nabla+Q$, where $Q$ is $4 \times 4$-matrix-valued and of order $O\left(|\mathbf{x}|^{-1}\right)$ at infinity. The approach is based on inversion with respect to the unit sphere in $\mathbb{R}^{3}$ and establishing embedding theorems for Dirac-Sobolev spaces of spinors $f$ which are such that $f$ and $H f$ lie in $\left(L^{p}\left(\mathbb{R}^{3}\right)\right)^{4}, 1 \leq$ $p<\infty$.
\end{abstract}

\section{INTRODUCTION}

The mathematical interpretation of the stability of matter problem concerns the question of whether the energy of a system of particles is bounded from below (stability of the first kind) and by a constant multiple of the number of particles (stability of the second kind). Dyson and Lenard made the initial breakthrough in 1967 for a non-relativistic model, and since then the problem has attracted a lot of attention, various relativistic models having been intensively studied in recent years. As was demonstrated by Fröhlich, Lieb and Loss in [3], to establish stability, it is of crucial importance to know if the kinetic energy operator has zero modes, i.e. eigenvectors corresponding to an eigenvalue at 0 ; the possibility that zero modes can exist was established at about the same time by Loss and Yau in [5]. Subsequently, Balinsky and Evans showed in [1] that zero modes are rare for these problems.

The first objective of the research reported here was to confirm that the results in [1] can be extended to Dirac-type operators with matrixvalued potentials. This then set the scene for the main goal which was to determine the decay rates of zero modes whenever they occur. The Dirac operator considered is of the form,

$$
H=\alpha \cdot \mathbf{p}+Q, \quad \mathbf{p}=-i \nabla
$$

where $\alpha$ is the triple of Dirac matrices and $Q$ is a $4 \times 4$ matrix-valued function. Assuming that $\|Q(\cdot)\|_{\mathbb{C}^{4}} \in L^{3}\left(\mathbb{R}^{3}\right)$, where $\|\cdot\|_{\mathbb{C}^{4}}$ denotes any matrix norm on $\mathbb{C}^{4}$, it was shown that $Q$ is a small perturbation of $\alpha \cdot \mathbf{p}$ and hence (1.1) defines a self-adjoint operator $H$ as an operator

Date: April 25, 2022.

The authors gratefully acknowledge the support of the EPSRC under grant $\mathrm{EP} / \mathrm{E} 04834 \mathrm{X} / 1$. 
sum with domain $\left(H^{1,2}\left(\mathbb{R}^{3}\right)\right)^{4}$, the space of 4 -component spinors in $\left(L^{2}\left(\mathbb{R}^{3}\right)\right)^{4}$ with weak first derivatives in $\left(L^{2}\left(\mathbb{R}^{3}\right)\right)^{4}$. The technique in [1] readily applied to (1.1) to meet the first objective and yield the following result:

Theorem 1. Let $\|Q(\cdot)\|_{\mathbb{C}^{4}} \in L^{3}\left(\mathbb{R}^{3}\right)$. Then $H_{t}:=\alpha \cdot \mathbf{p}+t Q, t \in R^{+}$ can have a zero mode for only a countable set of values of $t$. Moreover

$$
\operatorname{nul}(\mathrm{H}) \leq \text { const. } \int_{\mathbb{R}^{3}}|\mathrm{Q}(\mathbf{x})|^{3} \mathrm{~d} \mathbf{x},
$$

where $\operatorname{nul}(\mathrm{H})$ denotes the nullity of $H$, i.e. the dimension of the kernel of $H$.

It is proved in [6] (see also [7]) that if $\left.\|Q(\mathbf{x})\|_{\mathbb{C}^{4}}=O\left(\langle\mathbf{x}\rangle^{-\rho}\right), \rho\right\rangle 1$, where $\langle\mathbf{x}\rangle:=\sqrt{1+|\mathbf{x}|^{2}}$, then any zero mode is $O\left(|\mathbf{x}|^{-2}\right)$ at $\infty$ and there are no resonances (defined as a solution $\psi$ say which is such that $<\mathbf{x}>^{-s} \psi \in\left(L^{2}\left(\mathbb{R}^{3}\right)\right)^{4}$ for some $\left.s>0\right)$ if $\rho>3 / 2$ : note that such a $Q$ satisfies the assumption of Theorem 1. In view of this result we concentrate our attention on more singular potentials $Q$, namely ones which satisfy

$$
\|Q(\mathbf{x})\|_{\mathbb{C}^{4}}=O\left(|\mathbf{x}|^{-1}\right), \quad \mathbf{x} \in B_{1}^{c}
$$

where $B_{1}$ is the unit ball, centre the origin in $\mathbb{R}^{3}$, and $B_{1}^{c}$ denotes its complement. We actually consider weak solutions of $H \psi=0$ in $\left(L^{2}\left(B_{1}^{c}\right)\right)^{4}$. Hence the behaviour of $Q$ at the origin, and indeed within $B_{1}$, does not feature. Our main result is given in Theorem 4 below. Our approach is very different to that in [6] and is based on two techniques. In the first we use inversion with respect to $B_{1}$ to replace the problem in $B_{1}^{c}$ by an analogous one in $B_{1}$. The second part involves establishing Sobolev-type embedding theorems for the spaces $\mathbb{H}^{1, p}(\Omega)$ defined as the completion of $\left[C_{0}^{\infty}(\Omega)\right]^{4}$ with respect to the norm

$$
\|f\|_{1, p ; \Omega}:=\left\{\int_{\Omega}\left(|(\alpha \cdot \mathbf{p}) f|^{p}+|f|^{p}\right) d \mathbf{x}\right\}^{1 / p} .
$$

For $p=2$ these are just vector versions of the standard Sobolev spaces, but we need cases $p \neq 2$ for which we were unable to find any appropriate results in the literature. The results we give in section 3 use a method of Ledoux in [4] to derive a weak inequality and then embedding properties of Lorentz spaces on bounded domains.

\section{REDUCTION BY INVERSION}

We recall that in (1.1), $\alpha=\left(\alpha_{1}, \alpha_{2}, \alpha_{\mathbf{3}}\right)$ are Hermitian $4 \times 4$ matrices satisfying

$$
\alpha_{j} \alpha_{k}+\alpha_{k} \alpha_{j}=2 \delta_{j k} I_{4}, \quad j, k=1,2,3,
$$


where $I_{4}$ is the unit $4 \times 4$ matrix, and we choose

$$
\alpha_{j}=\left(\begin{array}{cc}
0_{2} & \sigma_{j} \\
\sigma_{j} & 0_{2}
\end{array}\right), \quad j=1,2,3
$$

where the $\sigma_{j}$ are the Pauli matrices

$$
\sigma_{1}=\left(\begin{array}{ll}
0 & 1 \\
1 & 0
\end{array}\right), \quad \sigma_{2}=\left(\begin{array}{ll}
0 & -i \\
i & 0
\end{array}\right), \quad \sigma_{3}=\left(\begin{array}{ll}
1 & 0 \\
0 & -1
\end{array}\right)
$$

and $0_{2}$ is the $2 \times 2$ zero matrix. From (1.2) we have that the $4 \times 4$ matrix-valued function $Q$ has components $q_{j k}, j, k=1, \cdots, 4$ which satisfy

$$
\left|q_{j k}(\mathbf{x})\right| \leq C|\mathbf{x}|^{-1}, \quad|\mathbf{x}| \geq 1 .
$$

For $\mathbb{C}^{4}$-valued functions $f, g$, measurable on an open set $\Omega \subseteq \mathbb{R}^{3}$, and $z, w \in \mathbb{C}^{4}$, we shall use the following notation:

$$
\begin{aligned}
<z, w> & :=\sum_{j=1}^{4} z_{j} \overline{w_{j}}, \quad|z|:=<z, z>^{\frac{1}{2}} \\
(f, g)_{\Omega} & :=\int_{\Omega}<f(\mathbf{x}), g(\mathbf{x})>d \mathbf{x} \\
\|f\|_{p, \Omega} & :=\left(\int_{\Omega}|f(x)|^{p} d \mathbf{x}\right)^{1 / p} .
\end{aligned}
$$

Thus $(f, g)_{\Omega}$ is the standard inner-product on $\left(L^{2}(\Omega)\right)^{4}$ and $\|f\|_{\Omega}=$ $\|f\|_{2, \Omega}$ the standard norm: when $\Omega=\mathbb{R}^{3}$ we shall simply write $(f, g)$ and $\|f\|$.

Let $\psi$ be a weak solution of $H \psi=0$ in $\mathbb{R}^{3} \backslash \bar{B}_{1}$, where $B_{1}$ is the open unit ball centre the origin and $\bar{B}_{1}$ is its closure: hence for all $\phi \in\left(C_{0}^{\infty}\left(\mathbb{R}^{3} \backslash \bar{B}_{1}\right)\right)^{4}$

$$
I:=\int<H \psi(\mathbf{x}), \phi(\mathbf{x})>d \mathbf{x}=0 .
$$

Inversion with respect to $B_{1}$ is the involution $\operatorname{Inv}: \mathbf{x} \mapsto \mathbf{y}, \mathbf{y}=\mathbf{x} /|\mathbf{x}|^{2}$, and for any function defined on $\mathbb{R}^{3} \backslash B_{1}$, the map $M: \phi \mapsto \tilde{\phi}:=$ $\phi \circ \operatorname{Inv}^{-1}$ is such that $\tilde{\phi}(\mathbf{y})=\phi(\mathbf{x})$ and yields a function on $B_{1}$. Hence $\phi \in\left(C_{0}^{\infty}\left(\mathbb{R}^{3} \backslash \bar{B}_{1}\right)\right)^{4}$ means that $\tilde{\phi} \in\left(C_{0}^{\infty}\left(B_{1} \backslash\{0\}\right)\right)^{4}$. The inversion gives

$$
M\{(\alpha \cdot \mathbf{p}) \psi\}(\mathbf{y})=|\mathbf{y}|^{2}(\beta \cdot \mathbf{p}) \tilde{\psi}(\mathbf{y}),
$$

where $\beta=\left(\beta_{1}, \beta_{2}, \beta_{3}\right)$ and

$$
\beta_{k}(\mathbf{y})=\sum_{j=1}^{3} \alpha_{j}\left(\delta_{j}^{k}-\frac{2 y_{k} y_{j}}{|\mathbf{y}|^{2}}\right),
$$


where $\delta_{j}^{k}$ is the Kronecker delta function. It is readily verified that the matrices $\beta_{k}(\mathbf{y})$ are Hermitian and satisfy

$$
\beta_{k}(\mathbf{y}) \beta_{j}(\mathbf{y})+\beta_{j}(\mathbf{y}) \beta_{k}(\mathbf{y})=2 \delta_{j}^{k} I_{4} .
$$

Also there exists a unitary matrix $X(\mathbf{y})$ such that $X \in C^{\infty}\left(\mathbb{R}^{3} \backslash\{0\}\right)$ and for all $\mathbf{y} \neq 0$,

$$
X(\mathbf{y})^{-1} \beta_{k}(\mathbf{y}) X(\mathbf{y})=-\alpha_{k}, \quad k=1,2,3 .
$$

Setting $\omega:=\mathbf{y} /|\mathbf{y}|$ it is easy to verify that these conditions are satisfied by

$$
X(\mathbf{y})=\left(\begin{array}{ll}
X_{2}(\mathbf{y}) & O_{2} \\
O_{2} & X_{2}(\mathbf{y})
\end{array}\right)
$$

where

$$
X(\mathbf{y})=\left(\begin{array}{ll}
i \omega_{3} & \omega_{2}+i \omega_{1} \\
-\omega_{2}+i \omega_{1} & -i \omega_{3}
\end{array}\right)
$$

Let $\tilde{\psi}(\mathbf{y})=-X(\mathbf{y}) \Psi(\mathbf{y})$. Then from (2.4) we have

$$
M\{(\alpha \cdot \mathbf{p}) \psi\}(\mathbf{y})=|\mathbf{y}|^{2} X(\mathbf{y})\{(\alpha \cdot \mathbf{p}) \Psi(\mathbf{y})+Y(\mathbf{y}) \Psi(\mathbf{y})\},
$$

where

$$
Y(\mathbf{y})=\sum_{k=1}^{3} \alpha_{k} X(y)^{-1}\left(-i \frac{\partial}{\partial_{y_{k}}} X(\mathbf{y})\right) .
$$

Also, a calculation gives that the Jacobian of the inversion gives $d \mathbf{x}=$ $|\mathbf{y}|^{-6} d \mathbf{y}$.

Returning now to (2.3), and with $\tilde{\phi}(\mathbf{y})=-X(\mathbf{y}) \Phi(\mathbf{y})$, the inversion yields

$$
\begin{aligned}
I & =\int_{B_{1}}<|\mathbf{y}|^{2} X(\mathbf{y})\{(\alpha \cdot \mathbf{p}) \Psi(\mathbf{y})+Y(\mathbf{y}) \Psi(\mathbf{y}) \\
& -\tilde{Q}(\mathbf{y}) X(\mathbf{y}) \Psi(\mathbf{y})\}, X(\mathbf{y}) \Phi(\mathbf{y})>|\mathbf{y}|^{-6} d \mathbf{y}=0
\end{aligned}
$$

for all $\Phi \in\left(C_{0}^{\infty}\left(B_{1} \backslash\{0\}\right)\right)^{4}$, which can be written as

$$
I=\int_{B_{1}}<(\alpha \cdot \mathbf{p}) \Psi(\mathbf{y})+Z(\mathbf{y}) \Psi(\mathbf{y}),|\mathbf{y}|^{-4} \Phi(\mathbf{y})>d \mathbf{y}=0
$$

where

$$
Z(\mathbf{y})=Y(\mathbf{y})-|\mathbf{y}|^{-2} X(\mathbf{y})^{-1} \tilde{Q}(\mathbf{y}) X(\mathbf{y}) .
$$

Equivalently, we can remove the factor $|\mathbf{y}|^{-4}$ in (2.9) to give

$$
I=\int_{B_{1}}<(\alpha \cdot \mathbf{p}) \Psi(\mathbf{y})+Z(\mathbf{y}) \Psi(\mathbf{y}), \Phi(\mathbf{y})>d \mathbf{y}=0
$$

for all $\Phi \in\left(C_{0}^{\infty}\left(B_{1} \backslash\{0\}\right)\right)^{4}$.

Let $\zeta \in C^{\infty}\left(\mathbb{R}^{+}\right)$satisfy

$$
\zeta(t)= \begin{cases}0 & \text { for } 0<t<1 \\ 1 & \text { for } t>2\end{cases}
$$


and for $\mathbf{y} \in \mathbb{R}^{3}$ set $\zeta_{n}(\mathbf{y})=\zeta(n|\mathbf{y}|)$. Then

$$
\nabla \zeta_{n}(\mathbf{y})=n \zeta^{\prime}(n|\mathbf{y}|) \frac{\mathbf{y}}{|\mathbf{y}|}
$$

and so

$$
\left|\nabla \zeta_{n}(\mathbf{y})\right|=O\left(n \chi_{\left[\frac{1}{n}, \frac{2}{n}\right]}(|\mathbf{y}|)\right)
$$

where $\chi_{I}$ denotes the characteristic function of the interval $I$. We then have from (2.11), now for all $\Phi \in\left(C_{0}^{\infty}\left(B_{1}\right)\right)^{4}$,

$$
\begin{aligned}
I & =\int_{B_{1}}<(\alpha \cdot \mathbf{p}) \Psi(\mathbf{y})+Z(\mathbf{y}) \Psi(\mathbf{y}), \zeta_{n}(\mathbf{y}) \Phi(\mathbf{y})>d \mathbf{y} \\
& =\int_{B_{1}}<\zeta_{n}(\mathbf{y})\{(\alpha \cdot \mathbf{p}) \Psi(\mathbf{y})+Z(\mathbf{y}) \Psi(\mathbf{y})\}, \Phi(\mathbf{y})>d \mathbf{y} \\
& =0 .
\end{aligned}
$$

In $I$,

$$
\zeta_{n}(\mathbf{y})(\alpha \cdot \mathbf{p}) \Psi(\mathbf{y})=(\alpha \cdot \mathbf{p})\left(\zeta_{n} \Psi\right)(\mathbf{y})-\left[(\alpha \cdot \mathbf{p}) \zeta_{n}\right](\Psi)(\mathbf{y})
$$

and

$$
V_{n}(\mathbf{y}):=\left[(\alpha \cdot \mathbf{p}) \zeta_{n}\right]=O\left(n \chi_{\left[\frac{1}{n}, \frac{2}{n}\right]}\right)
$$

Therefore, as $n \rightarrow \infty$,

$$
\begin{aligned}
\left|\int_{B_{1}}<V_{n}(\mathbf{y}) \Phi(\mathbf{y}), \Phi(\mathbf{y})>d \mathbf{y}\right| & \leq n\|\Psi\|_{L^{\infty}\left(B_{1}\right)} \int_{B_{1}} \chi_{\left[\frac{1}{n}, \frac{2}{n}\right]} d \mathbf{y} \\
& =O\left(n^{-2}\right) \rightarrow 0 .
\end{aligned}
$$

Also

$$
\begin{aligned}
& \int_{B_{1}}<[(\alpha \cdot \mathbf{p})+Z(\mathbf{y})] \zeta_{n}(\mathbf{y}) \Psi(\mathbf{y}), \Phi(\mathbf{y})>d \mathbf{y} \\
& =\int_{B_{1}}<\zeta_{n}(\mathbf{y}) \Psi(\mathbf{y}),[(\alpha \cdot \mathbf{p})+Z(\mathbf{y})] \Phi(\mathbf{y})>d \mathbf{y} \\
& \rightarrow \int_{B_{1}}<\Psi(\mathbf{y}),[(\alpha \cdot \mathbf{p})+Z(\mathbf{y})] \Phi(\mathbf{y})>d \mathbf{y} .
\end{aligned}
$$

We have therefore proved that

$$
\int_{B_{1}}<[(\alpha \cdot \mathbf{p})+Z(\mathbf{y})] \Psi(\mathbf{y}), \Phi(\mathbf{y})>d \mathbf{y}=0
$$

for all $\Phi \in\left(C_{0}^{\infty}\left(B_{1}\right)\right)^{4}$. In other words

$$
[(\alpha \cdot \mathbf{p})+Z(\mathbf{y})] \Psi(\mathbf{y})=0
$$

in the weak sense. From (2.10) it follows that

$$
\|Z(\mathbf{y})\|_{\mathbb{C}^{4}} \leq C|\mathbf{y}|^{-1} .
$$




\section{Dirac-Sobolev inequalities}

Let $\mathbb{H}^{1, p}(\Omega), 1 \leq p<\infty$, denote the completion of $\left[C_{0}^{\infty}(\Omega)\right]^{4}$ with respect to the norm

$$
\|f\|_{1, p ; \Omega}:=\left\{\int_{\Omega}\left(|(\alpha \cdot \mathbf{p}) f|^{p}+|f|^{p}\right) d \mathbf{x}\right\}^{1 / p} .
$$

We also use the notation

$$
\mathbb{D}:=(\alpha \cdot \mathbf{p})^{2}, \quad \mathbb{P}_{t}: e^{-t \mathbb{D}}, \quad t \geq 0 .
$$

Then,

$$
(\mathbb{D} f)_{j}=-\Delta f_{j}, \quad\left(\mathbb{P}_{t} f\right)_{j}=e^{-t \Delta} f_{j}, \quad j=1,2,3,4,
$$

where $\left\{e^{-t \Delta}\right\}_{t \geq 0}$ is the heat semigroup. Furthermore, for all $t>0, \mathbf{x} \in$ $\Omega$,

and so

$$
\left(e^{-t \Delta} f_{j}\right)(\mathbf{x})=\frac{1}{(4 \pi t)^{3 / 2}} \int_{\mathbb{R}^{3}} f_{j}(\mathbf{y}) e^{-|\mathbf{x}-\mathbf{y}|^{2} / 4 t} d \mathbf{y},
$$

$$
\left(\mathbb{P}_{t} f\right)(\mathbf{x})=\frac{1}{(4 \pi t)^{3 / 2}} \int_{\mathbb{R}^{3}} f(\mathbf{y}) e^{-|\mathbf{x}-\mathbf{y}|^{2} / 4 t} d \mathbf{y} .
$$

Note that if $\Omega \neq \mathbb{R}^{n}$ we put any $f \in \mathbb{H}^{1, p}(\Omega)$ to be zero outside $\Omega$ and hence is in $\mathbb{H}^{1, p} \equiv \mathbb{H}^{1, p}\left(\mathbb{R}^{n}\right)$. Define

$$
\|f\|_{B^{\alpha}(\Omega)}:=\sup _{t>0}\left\{t^{-\alpha / 2}\left|\mathbb{P}_{t} f\right|_{\infty ; \Omega}\right\}
$$

where $\left|\mathbb{P}_{t} f\right|_{\infty ; \Omega}:=\sup _{\mathbf{x} \in \Omega}\left|\mathbb{P}_{t} f(\mathbf{x})\right|$, and denote by $B^{\alpha}(\Omega)$ the completion of $\left[C_{0}^{\infty}(\Omega)\right]^{4}$ with respect to $\|\cdot\|_{B^{\alpha}(\Omega)}$.

Our main theorem in this section introduces the weak- $L^{q}$ space on $\Omega$, written, $L^{q, \infty}(\Omega)$, which is defined by

$$
\|f\|_{q, \infty ; \Omega}: \sup _{u>0}\left\{u^{q} \lambda(|f| \geq u)\right\},
$$

where $\lambda$ denotes Lebesgue measure and $\lambda(|f| \geq u)$ stands for the measure of the set in $\Omega$ on which $|f(\mathbf{x})| \geq u$.

Theorem 2. Let $1 \leq p<q<\infty$ and let $f$ be such that $\|(\alpha \cdot \mathbf{p}) f\|_{p, \Omega}<$ $\infty$ and $f \in B^{\theta /(\theta-1)}(\Omega)$, for $\theta=p / q$. Then we have for some constant $C>0$,

$$
\|f\|_{q, \infty ; \Omega} \leq C\|(\alpha \cdot \mathbf{p}) f\|_{p, \Omega}^{\theta}\|f\|_{B^{\theta /(\theta-1)}(\Omega)}^{1-\theta} .
$$

Proof. For simplicity of notation, we suppress the dependence of the norms and spaces on $\Omega$ throughout the proof. It is sufficient to prove the result for $f \in\left(C_{0}^{\infty}(\Omega)\right)^{4}$. The proof is inspired by that of Ledoux in [4]. By homogeneity, we may assume that $\|f\|_{B^{\theta /(\theta-1)}} \leq 1$, and so

$$
\left|\mathbb{P}_{t} f\right|_{\infty} \leq t^{\theta / 2(\theta-1)}
$$

for all $t>0$. Thus, on choosing $t_{u}=u^{2(\theta-1) / \theta}$ it follows that

$$
\left|\mathbb{P}_{t_{u}} f\right|_{\infty} \leq u \text {. }
$$


This gives that $|f| \geq 2 u$ implies that $\left|f-\mathbb{P}_{t_{u}} f\right| \geq|f|-\left|\mathbb{P}_{t} f\right| \geq u$ and consequently

$$
\begin{aligned}
u^{q} \lambda(|f| \geq 2 u) & \leq u^{q} \lambda\left(\left|f-\mathbb{P}_{t_{u}}\right| \geq u\right) \\
& \leq u^{q-p} \int\left|f-\mathbb{P}_{t_{u}}\right|^{p} d \mathbf{x} .
\end{aligned}
$$

Since

$$
\frac{\partial}{\partial t} \mathbb{P}_{t} f=(\alpha \cdot \mathbf{p})^{2} \mathbb{P}_{t} f, \quad \mathbb{P}_{0} f=f,
$$

in view of the analogous result for $e^{-t \Delta}$ on each component of $f$, it follows that

$$
\mathbb{P}_{t} f-f=\int_{0}^{t}(\alpha \cdot \mathbf{p})^{2} \mathbb{P}_{s} f d s
$$

and hence for all $g \in\left[C_{0}^{\infty}\left(B_{1}\right)\right]^{4}$,

$$
\begin{aligned}
\int_{\mathbb{R}^{3}}<g, f-\mathbb{P}_{t} f>d \mathbf{x} & =-\int_{0}^{t}\left(\int_{\mathbb{R}^{3}}<g,(\alpha \cdot \mathbf{p})^{2} \mathbb{P}_{s} f>d \mathbf{x}\right) d s \\
& =-\int_{0}^{t}\left(\int_{\mathbb{R}^{3}}<(\alpha \cdot \mathbf{p}) \mathbb{P}_{s} g,(\alpha \cdot \mathbf{p}) f>d \mathbf{x}\right) d s \\
& \leq\|(\alpha \cdot \mathbf{p}) f\|_{p} \int_{0}^{t}\left\|(\alpha \cdot \mathbf{p}) \mathbb{P}_{s} g\right\|_{p^{\prime}} d s,
\end{aligned}
$$

where $p^{\prime}=p /(p-1)$ for $p>1$ and $p^{\prime}=\infty$ otherwise. From (3.5) we have

$$
\begin{aligned}
(\alpha \cdot \mathbf{p}) \mathbb{P}_{s} g(\mathbf{x}) & =\frac{1}{(4 \pi s)^{3 / 2}} \sum_{j=1}^{3} \alpha_{j} \int_{\mathbb{R}^{3}} g(\mathbf{y})\left(-i \frac{\partial}{\partial x_{j}}\right) e^{-\frac{|\mathbf{x}-\mathbf{y}|^{2}}{4 s}} d \mathbf{y} \\
& =\frac{i}{(4 \pi s)^{3 / 2}} \frac{1}{2 s} \int_{\mathbb{R}^{3}} e^{-\frac{|\mathbf{x}-\mathbf{y}|^{2}}{4 s}}[\alpha \cdot(\mathbf{x}-\mathbf{y})] g(\mathbf{y}) d \mathbf{y} .
\end{aligned}
$$

On using Young's inequality for convolutions, this yields

$$
\begin{aligned}
\left\|(\alpha \cdot \mathbf{p}) \mathbb{P}_{s} g\right\|_{p^{\prime}} & \leq \frac{1}{(4 \pi s)^{3 / 2}} \frac{1}{2 s} \int_{\mathbb{R}^{3}}[\alpha \cdot \mathbf{z}] e^{-\frac{|\mathbf{z}|^{2}}{4 s}} d \mathbf{z}\|g\|_{p^{\prime}} \\
& \leq C s^{-\frac{1}{2}}\|g\|_{p^{\prime}}
\end{aligned}
$$

for all $p \in[1, \infty)$. We therefore have

$$
\left|\int<g, f-\mathbb{P}_{t} f>d \mathbf{x}\right| \leq C t^{\frac{1}{2}}\|(\alpha \cdot \mathbf{p}) f\|_{p}\|g\|_{p^{\prime}}
$$

and thus

$$
\left\|f-\mathbb{P}_{t} f\right\|_{p} \leq C t^{\frac{1}{2}}\|(\alpha \cdot \mathbf{p}) f\|_{p} .
$$

On substituting this in (3.8) we have

$$
\begin{aligned}
u^{q} \lambda(|f| \geq 2 u) & \leq C u^{q-p} t_{u}^{p / 2}\|(\alpha \cdot \mathbf{p}) f\|_{p}^{p} \\
& =C\|(\alpha \cdot \mathbf{p}) f\|_{p}^{p}
\end{aligned}
$$


since $q-p+p(\theta-1) / \theta=0$, whence the result.

In the following corollary, the notation indicates that integration is over $B_{1}$.

Corollary 1. Let $1 \leq p<q<\infty, r:=3\left(\frac{q}{p}-1\right) \in[1, p]$ and $f \in$ $\mathbb{H}^{1, p}\left(B_{1}\right)$. Then we have that for any $k \in(0, q)$ and $\theta=p / q$, there exists a positive constant $C$ such that

$$
\|f\|_{k, B_{1}} \leq C\|(\alpha \cdot \mathbf{p}) f\|_{p, B_{1}}^{\theta}\|f\|_{r, B_{1}}^{1-\theta}
$$

Proof. All norms in the proof are over $B_{1}$. From (3.5), for any $r \in[1, \infty]$ and with $r^{\prime}=r /(r-1), r>1$,

$$
\begin{aligned}
\left|\mathbb{P}_{t} f(\mathbf{x})\right| & \leq \frac{1}{(4 \pi t)^{\frac{3}{2}}}\|f\|_{r}\left(\int_{B_{1}} e^{-r^{\prime}|\mathbf{x}-\mathbf{y}|^{2} / 4 t} d \mathbf{y}\right)^{1 / r^{\prime}} \\
& \leq C t^{-3 / 2 r}\|f\|_{r}
\end{aligned}
$$

note that this holds also for $r=1$. Hence,

$$
\begin{aligned}
\|f\|_{B^{\theta /(\theta-1)}} & \leq C \sup _{t>0} t^{-\frac{\theta}{2(\theta-1)}-\frac{3}{2 r}}\|f\|_{r} \\
& =C\|f\|_{r}
\end{aligned}
$$

if $\frac{\theta}{2(\theta-1)}+\frac{3}{2 r}=0$, which is true if $r=3(q / p-1)$ since $\theta=p / q$. Since $r \leq p$ and $f \in L^{p}\left(B_{1}\right)$ it follows that $f \in L^{r}\left(B_{1}\right)$ and hence $f \in B^{\theta /(\theta-1)}$. From (3.6) we therefore have

$$
\|f\|_{q, \infty} \leq C\|(\alpha \cdot \mathbf{p}) f\|_{p}^{\theta}\|f\|_{r}^{(1-\theta)} .
$$

But for Lorentz spaces $L^{r, s}$ on a set $\Omega$ of finite measure, we have the continuous embeddings (see [2], Proposition 3.4.4)

$$
L^{q, s}(\Omega) \hookrightarrow L^{k, m}
$$

if $0<k<q \leq \infty, 0<s, m \leq \infty$. In particular, with $s=\infty, m=k$, and recalling that $L^{k, k}=L^{k}$, we have for $0<k<q \leq \infty$,

$$
L^{q, \infty} \hookrightarrow L^{k}
$$

and so

$$
\|f\|_{k} \leq C\|f\|_{q, \infty} .
$$

The corollary follows from (3.11).

Corollary 2. Let $p \in[1, \infty), k \in[1, p(p+3) / 3)$ and $f \in \mathbb{H}^{1, p}\left(B_{1}\right)$. Then there exists a positive constant $C$ such that

$$
\|f\|_{k, B_{1}} \leq C\|(\alpha \cdot \mathbf{p}) f\|_{p, B_{1}} .
$$


Proof. For $k \in[1, p]$, we choose in Corollary $1, q=p(k+3) / 3$ and $r=k$ to deduce (3.13) from (3.10). When $k \in(p, 4 p / 3)$ we choose any $q \in[4 p / 3, p(p+3) / 3]$, so that $q>k>p$ and $r=3(q / p-1) \in[1, p]$. When $k \in[4 p / 3, p(p+3) / 3)$ we choose any $q \in(k, p(p+3) / 3]$ so that $q>k>p$ and $r \in(1, p]$. In both the last two cases we also have $r<k$ and hence

$$
\|f\|_{r, B_{1}} \leq C\|f\|_{k, B_{1}} .
$$

This yields (3.13) from (3.10).

\section{Estimate FOR ZERO MODES}

Let $\psi$ be such that $\psi,(\alpha \cdot \mathbf{p}) \psi \in L^{2}\left(B_{1}^{c}\right), B_{1}^{c}:=\mathbb{R}^{3} \backslash B_{1}$, and

$$
(\alpha \cdot \mathbf{p}) \psi(\mathbf{x})=-Q(\mathbf{x}) \psi(\mathbf{x})
$$

where

$$
\|Q(\mathbf{x})\|_{\mathbb{C}^{4}}=O\left(|\mathbf{x}|^{-1}\right)
$$

and

$$
\psi \in\left(L^{2}\left(B_{1}^{c}\right)\right)^{4} .
$$

If $\theta \in C^{1}\left(\mathbb{R}^{3}\right)$ is 1 in the neighbourhood of $\infty$ and is supported in $\mathbb{R}^{3} \backslash \overline{B_{1}}$ then $\theta \psi$ has similar properties to those above. Hence we may assume, without loss of generality, that $\psi$ is supported in $\mathbb{R}^{3} \backslash \overline{B_{1}}$. Moreover,

$$
\int_{B_{1}^{c}}|\mathbf{x}|^{2}|(\alpha \cdot \mathbf{p}) \psi(\mathbf{x})|^{2} d \mathbf{x}<\infty .
$$

On applying the inversion described in section 1, and using the notation

$$
(M \psi)(\mathbf{y})=: \tilde{\psi}(\mathbf{y})=:-X(\mathbf{y}) \Psi(\mathbf{y})
$$

where $|X(\mathbf{y})| \asymp 1$, we have from $\psi \in L^{2}\left(\mathbf{B}_{1}^{c}\right)$ that

$$
\int_{B_{1}}|\Psi(\mathbf{y})|^{2} \frac{d \mathbf{y}}{|\mathbf{y}|^{6}}<\infty
$$

We also have from (2.7) that

$$
(M(\alpha \cdot \mathbf{p}) \psi)(\mathbf{y})=|\mathbf{y}|^{2}\{X(\mathbf{y})[(\alpha \cdot \mathbf{p}) \Psi(\mathbf{y})+Y(\mathbf{y}) \Psi(\mathbf{y})]\},
$$

where $Y(\mathbf{y})$ is given in (2.8) and is readily seen to satisfy

$$
\|Y(\mathbf{y})\|_{\mathbb{C}^{4}} \asymp 1 /|\mathbf{y}| \text {. }
$$

Let $\Psi(\mathbf{y})=|\mathbf{y}|^{2} \Phi(\mathbf{y})$. Then

$$
(\alpha \cdot \mathbf{p}) \Psi(\mathbf{y})=|\mathbf{y}|^{2}\left\{(\alpha \cdot \mathbf{p}) \Phi(\mathbf{y})+O\left(\frac{|\Phi(\mathbf{y})|}{|\mathbf{y}|}\right)\right\}
$$

and so from (4.4)

$$
|M(\alpha \cdot \mathbf{p}) \psi(\mathbf{y})| \asymp|\mathbf{y}|^{4}\left\{(\alpha \cdot \mathbf{p}) \Phi(\mathbf{y})+O\left(\frac{|\Phi(\mathbf{y})|}{|\mathbf{y}|}\right)\right\} .
$$


Hence from (4.1)

$$
\left.\left.\int_{B_{1}}|\mathbf{y}|^{-2}|| \mathbf{y}\right|^{4}\left\{(\alpha \cdot \mathbf{p}) \Phi(\mathbf{y})+O\left(\frac{|\Phi(\mathbf{y})|}{|\mathbf{y}|}\right)\right\}\right|^{2} \frac{d \mathbf{y}}{|\mathbf{y}|^{6}}<\infty
$$

Since, from (4.3),

$$
\int_{B_{1}}|\Phi(\mathbf{y})|^{2} \frac{d \mathbf{y}}{|\mathbf{y}|^{2}}<\infty
$$

it follows from (4.6) that

$$
\int_{B_{1}}|(\alpha \cdot \mathbf{p}) \Phi(\mathbf{y})|^{2} d y<\infty .
$$

Hence, $\Phi \in \mathbb{H}^{1,2}\left(B_{1}\right)$ : recall that we may assume that $\psi$ is supported in $\mathbb{R}^{3} \backslash \overline{B_{1}}$ and hence $\Phi$ is supported in $B_{1}$. By Corollary 2, we have that $\Phi \in L^{k}\left(B_{1}\right)$ for any $k \in[1,10 / 3)$.

We therefore have the preliminary result

Theorem 3. Let $\psi \in L^{2}\left(B_{1}^{c}\right)$ be a solution of 4.1) with $Q(\mathbf{x})=$ $O\left(|\mathbf{x}|^{-1}\right)$ in $B_{1}^{c}$. Then, for any $k \in[1,10 / 3)$,

$$
\psi(\mathbf{x})=|\mathbf{x}|^{-2} \phi(\mathbf{x})
$$

where

$$
\int_{B_{1}^{c}}|\phi(\mathbf{x})|^{k}|\mathbf{x}|^{-6} d \mathbf{x}<\infty
$$

However, we can repeat the analysis that led to Theorem 3 to improve this result. From $\Psi(\mathbf{y})=|\mathbf{y}|^{2} \Phi(\mathbf{y})$ and (2.14) it follows that

$$
(\alpha \cdot \mathbf{p}) \Phi(\mathbf{y})=-Z^{(1)}(\mathbf{y}) \Phi(\mathbf{y})
$$

where

$$
Z^{(1)}=Z(\mathbf{y})-2 i|\mathbf{y}|^{-2}(\alpha \cdot \mathbf{y})=O\left(\frac{1}{|\mathbf{y}|}\right) .
$$

Let $\Phi(\mathbf{y})=|\mathbf{y}|^{t} \Phi^{(2)}(\mathbf{y})$. Then we have

$$
(\alpha \cdot \mathbf{p}) \Phi^{(2)}(\mathbf{y})=-Z^{(2)}(\mathbf{y}) \Phi^{(2)}(\mathbf{y}),
$$

where

$$
Z^{(2)}(\mathbf{y})=O\left(\frac{1}{|\mathbf{y}|}\right)
$$

By Hölder's inequality, for $1 \leq p<k, k \in[1,10 / 3)$,

$$
\begin{aligned}
\int_{B_{1}}\left|Z^{(2)}(\mathbf{y}) \Phi^{(2)}(\mathbf{y})\right|^{p} d \mathbf{y} & \leq\left.\left. C \int_{B_{1}}|| \mathbf{y}\right|^{-(1+t)} \Phi(\mathbf{y})\right|^{p} d \mathbf{y} \\
& \leq\left(\int_{B_{1}}|\Phi(\mathbf{y})|^{k} d \mathbf{y}\right)^{p / k}\left(\int_{B_{1}}|\mathbf{y}|^{-p(1+t)(k /(k-p)} d \mathbf{y}\right)^{1-p / k} \\
& <\infty
\end{aligned}
$$

if

$$
p\left(\frac{1+t}{3}+\frac{1}{k}\right)<1
$$


Choose $p=1$ and let $0<t<11 / 10$. Then (4.8) is satisfied for some $k \in[1,10 / 3)$, depending on $t$. We therefore have that $\Phi^{(2)} \in \mathbb{H}^{1,1}\left(B_{1}\right)$ if $0<t<11 / 10$, and hence, by Corollary 2 , that $\Phi^{(2)} \in L^{s}\left(B_{1}\right)$ for any $s \in[1,4 / 3)$. The following result has consequently been proved.

Theorem 4. Suppose that $\psi \in L^{2}\left(B_{1}^{c}\right)$ satisfies $\{(\alpha \cdot \mathbf{p})+Q(\mathbf{x})\} \psi(\mathbf{x})=$ 0 in $B_{1}^{c}$. Then for any $0<t<11 / 10, \psi(\mathbf{x})=|\mathbf{x}|^{-2-t} \phi(\mathbf{x})$, where

$$
\int_{B_{1}^{c}}|\phi(\mathbf{x})|^{s}|\mathbf{x}|^{-6} d \mathbf{x}<\infty
$$

for any $s \in[1,4 / 3)$.

\section{REFERENCES}

[1] A. Balinsky and W. D. Evans, On the zero modes of Weyl-Dirac operators and their multiplicity. Bull.L.M.S. 34 (2002), 236-242.

[2] D. E. Edmunds and W. D. Evans, Hardy Operators, Function Spaces and Embeddings.Springer: Berlin, Heidelberg, New York, 2004.

[3] J. Frölich, E. Lieb and M. Loss, Stability of Coulomb systems with magnetic fields, I: the one-electron atom. Comm. Math. Phys. 104 (1986) 251-270.

[4] M. Ledoux, On improved Sobolev embedding theorems, Math.Res. Lett. 10, 659-669 (2003).

[5] M. Loss and H-T Yau, Stability of Coulomb systems with magnetic fields, III: zero energy bound states of the Pauli operator. Comm. Math. Phys. 104 (1986) 283-290.

[6] Y. Saitō and T. Umeda, The zero modes and zero resonances of massless Dirac operators. To appear in Hokkaido Math.J..

[7] Y. Saitō and T. Umeda, The asymptotic limits of zero modes of massless Dirac operators. To appear in Letters of Math.Phys.

School of Mathematics, Cardiff University, 23 Senghennydd Road, CARDIFF CF2 4YH, UK

E-mail address: BalinskyA@cardiff.ac.uk

School of Mathematics, Cardiff University, 23 Senghennydd Road, CARDIFF CF2 4YH, UK

E-mail address: EvansWD@cardiff.ac.uk

Department of Mathematics, University of Alabama at Birmingham, BiRminghAM, AL 35294-1170, USA

E-mail address: saito@math.uab.edu 